\title{
FORO
}

Foro presenta opiniones disidentes dentro del campo de la biología y considera para publicación respuestas de nuestros(as) lectores (as).

Forum presents dissident opinions in the field of biology and considers for publication formal replies from our readers.

\section{Los felinos: ¿Una alternativa en estudios de toxicología genética?}

\author{
Ana Zamora-Perez ${ }^{1}$, Belinda C. Gómez-Meda², María L. Ramos-Ibarra ${ }^{3}$, Cecilia M. Batista-
} González ${ }^{4}$, Jaime Luna-Aguirre ${ }^{3}$, Andrés González-Rodríguez ${ }^{5}$, José L. Rodríguez-Ávila ${ }^{6}$ \& Guillermo M. Zúñiga-González ${ }^{4}$

1. Instituto de Investigación en Odontología, Centro Universitario de Ciencias de la Salud, Universidad de Guadalajara, Guadalajara, Jalisco, México. Phone: (33) 36170808, Fax: (33) 36171019; anazamora@gmail.com

2. Instituto de Biología Molecular en Medicina, Centro Universitario de Ciencias de la Salud, Universidad de Guadalajara, Guadalajara, Jalisco, México.

3. Centro Universitario de Ciencias Biológicas y Agropecuarias, Universidad de Guadalajara, Guadalajara, Jalisco, México.

4. Laboratorio de Mutagénesis, Centro de Investigación Biomédica de Occidente, Instituto Mexicano del Seguro Social, Guadalajara, Jalisco, México.

5. Centro para la Conservación e Investigación de la Vida Silvestre, Dirección General de Vida Silvestre, Secretaría del Medio Ambiente y Recursos Naturales, Guadalajara, Jalisco, México.

6. Zoológico Guadalajara y Zoológico Puerto Vallarta, Jalisco, México.

\section{Recibido 10-IV-2007. Corregido 10-III-2008. Aceptado 31-III-2008.}

\begin{abstract}
Felines: an alternative in genetic toxicology studies? The micronuclei (MN) test carry out in peripheral blood is fast, simple, economic and it is used to detect genotoxic environmental agents. MN are fragments of chromosomes or complete chromosomes remaining in the cytoplasm after cell division, which increase when organisms are exposed to genotoxic agents. Therefore, species with the highest values of spontaneous micronucleated erythrocytes (MNE) are the most suitable to be potentials biomonitor of micronucleogenic agents, using a drop of blood. Nine species of Felines that present spontaneous MNE in peripheral blood are shown. From these species, the cat has been previously proven, with positive results and also lion (Panthera leo), yaguaroundi (Felis yagoaroundi), lynx (Lynx ruffus), jaguar (Panthera onca), puma (Puma concolor), tiger (Panthera tigris), ocelote (Felis padalis) and leopard (Panthera pardus) display spontaneous MNE, and with this characteristic this Family can be propose like a potential group to be used in toxicogenetic studies. Rev. Biol. Trop. 56 (2): 969-974. Epub 2008 June 30.
\end{abstract}

Key words. Felines, micronuclei, biomonitor, genotoxicity, erythrocytes, peripheral blood.

\section{La prueba de micronúcleos}

Los micronúcleos $(\mathrm{MN})$ se están usando para estudiar genotoxicidad en algunas especies. Nuestro grupo ha estudiado a los micronúcleos espontáneos de gran cantidad de especies, con la intención de seleccionar aquellas con el mayor número para proponerlas como modelos para estudios de genotoxicidad (Zúñiga 1996a, Zúñiga-González 1998, Zúñiga-González 2000, Zúñiga-González 2001a, Zúñiga-González 2001, Zúñiga-González 2002). Los MN espontáneos no pueden ser observados en la sangre periférica de todas las especies y sólo aquellas que presentan esta característica pueden ser "útiles" como monitores de genotóxicos mediante la prueba de MN. En estas especies, el sistema que se encarga de retirarlos de la circulación no es muy eficiente y podemos verlos por acumulación en su sangre periférica (Zúñiga 1996a, Oxenham 1999, Zúñiga-González 2000, Zúñiga-González 2001) y en número significativamente mayor cuando estas especies se exponen a genotóxicos ambientales. 
Si bien ya se conocen especies que pueden ser utilizadas (en su mayoría de laboratorio), en toxicología genética se acepta que una sola prueba no permite concluir respecto a la genotoxicidad de un compuesto, ya que existen drogas o medicamentos que responden de manera especie-específica o tejido-específico (Ramírez-Muñoz et al. 1999a), por lo que se vuelve importante tener diferentes alternativas.

\section{¿Que son los micronúcleos?}

Los MN son fragmentos de cromosomas o cromosomas completos que quedan fuera del núcleo en mitosis (Schimid 1975, Heddle et al. 1978, Corazza et al. 1990, McDonald et al. 1998). Su formación se basa en que en anafase, cualquier fragmento cromosómico que no posea centrómero no podrá integrarse al núcleo, pues carece del elemento indispensable para orientarse en el huso acromático. Después de la telofase los cromosomas normales, así como los fragmentos que posean centrómero, darán origen a los núcleos de las células hijas regulares. Los elementos rezagados (fragmentos o cromosomas completos) quedarán incluidos en el citoplasma de las células hijas y una considerable proporción es transformada en uno o varios núcleos secundarios que son como regla mucho más pequeños que el núcleo principal y de ahí el nombre de MN (Schmid 1975, Yamamoto et al. 1980, Heddle et al. 1983, Afshari et al. 1994). Entonces, la presencia de MN se traduce en el ámbito celular como pérdida de ADN.

\section{¿Qué determina el ensayo de MN?}

La prueba de $\mathrm{MN}$ detecta tanto agentes que rompen cromosomas (clastogénicos) como los que dañan el huso mitótico (aneuploidogénicos) (Schmid 1975, Heddle et al. 1983) y se realiza en tejidos con rápida proliferación celular (Heddle et al. 1983, Schmezer et al. 1990). El ensayo de $\mathrm{MN}$ en eritrocitos de sangre periférica es entonces una alternativa altamente informativa para el monitoreo de genotóxicos, que no requiere del sacrificio de los organismos, además de ser rápida y económica. Por tanto, utilizar organismos "centinelas" o bioindicadores para la prueba de $\mathrm{MN}$, puede darnos también información del efecto de nuevos medicamentos de uso veterinario.

\section{Felinos muestreados}

En el presente mostramos solamente especies de felinos, con la intención de remarcar la importancia de esta familia como un grupo potencialmente adecuado para estudios de genotoxicidad por presentar eritrocitos micronucleados espontáneos (EMNe). Hasta el momento hemos tenido la oportunidad de muestrear 37 animales de nueve especies diferentes de felinos, los cuales han sido obtenidos del Zoológico Guadalajara, Zoológico Puerto Vallarta y colecciones privadas (Zúñiga 1996a, Zúñiga-González 1998, ZúñigaGonzález 2000, Zúñiga-González 2001a, ZúñigaGonzález 2001, Zúñiga-González 2002).

\section{Obtención de la muestra de sangre para la valoración de MN}

Para la determinación de los eritrocitos micronucleados (EMN) se obtuvo una gota de sangre con la que se hicieron dos extendidos sobre portaobjetos limpios y desengrasados, se dejaron secar a temperatura ambiente, se fijaron en etanol absoluto por 10 minutos, se tiñeron con naranja de acridina (Zúñiga-González 2003), que es un colorante específico para ácidos nucleicos y se contaron EMNe en 10000 eritrocitos, mediante observación de la muestra en un microscopio equipado con lámpara de epifluorescencia. En la Fig. 1 se puede observar un EMN típico

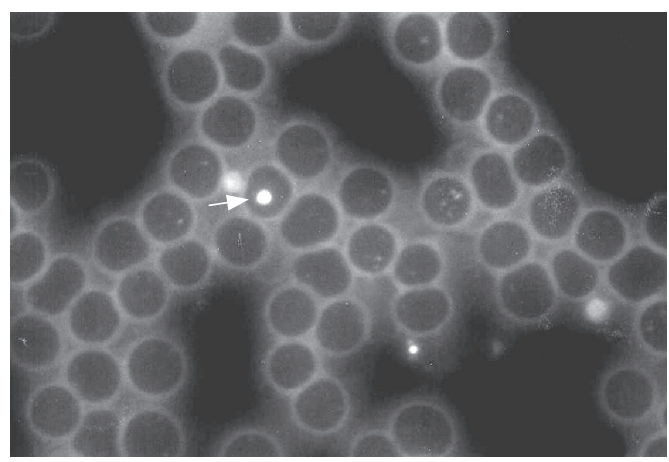

Fig. 1. La flecha indica un eritrocito de sangre periférica de felino con EMNe teñido con naranja de acridina. 
de sangre periférica de un felino. Los valores de EMNe de los felinos muestrados, se muestran en el Cuadro 1, como se puede observar, todas las especies mostraron EMNe, si bien, de una especie sólo se pudo obtener una muestra.

\section{El uso de biomonitores en toxicología genética y el ensayo de MN}

El monitoreo por el análisis directo de los agentes químicos requiere del conocimiento del contaminante a verificar y su evaluación está limitada por la sensibilidad y especificidad del método utilizado. En cambio, los bioindicadores nos permiten estudiar el agente problema, así como sus metabolitos, que en ocasiones pueden ser más tóxicos que el compuesto original (Rodríguez-Ariza 1992). Para el caso particular de la prueba de $\mathrm{MN}$, estos organismos deberán responder formando EMNe. Esto es debido a que la parte de su sistema inmunológico encargado de retirarlos, no tiene un estricto control sobre éstos y como consecuencia se pueden observar variaciones en sus valores en sangre periférica como resultado de su acumulación, por lo tanto, si a estos organismos se les expone a agentes genotóxicos, el número de EMN se incrementará por su acumulación (ZúñigaGonzález 1998, Zúñiga-González 2001a), con lo que se podrán detectar genotóxicos micronucleogénicos mediante la comparación de EMN de la sangre periférica antes y después de la exposición o al comparar los valores de poblaciones situadas en diferentes lugares.

Observamos que todas las especies muestreadas presentaron EMN, aunque, por estudios que hemos realizado con otras familias, sabemos que esta situación no es común. Por citar un ejemplo, en el orden de los Roedores existen especies que presentan EMN y especies que no los presentan dentro de la misma familia.

A simple vista se observa que el grupo de los felinos es bastante uniforme desde el punto de vista estructural y todas las especies que la conforman, desde el gato hasta el tigre tienen un enorme parecido "familiar", esto en otras familias no sucede y se requiere tener más conocimientos taxonómicos para poder relacionar especies

CUADRO 1

Eritrocitos micronucleados espontáneos de 9 especies de felinos

\begin{tabular}{|c|c|c|c|c|}
\hline Especie & $\mathrm{N}$ & Conteo individual de EMNe & Media \pm DE & Referencia \\
\hline León (Panthera leo) & 5 & $0.5,0.7,8.0,5.5,6.0$ & $4.1 \pm 3.4$ & $\begin{array}{l}\text { Zúñiga 1996a, Zúñiga- } \\
\text { González 2000, Zúñiga- } \\
\text { González 2001b }\end{array}$ \\
\hline Yaguaroundi (Felis yagoaroundi) & 3 & $4.5,9.0,7.0$ & $6.8 \pm 2.3$ & Zúñiga 1996a, \\
\hline Gato doméstico (Felis domesticus) & 6 & $6.0,8.0,8.0,6.5,13.0,9.0$ & $8.4 \pm 2.5$ & Zúñiga 1996a \\
\hline Gato siamés (Felis domesticus) & 3 & $11.0,11.0,10.0$ & $11.0 \pm 0.9$ & Zúñiga 1996a \\
\hline Lince (Lynx ruffus) & 4 & $1.0,12.0,19.0,15.0$ & $11.9 \pm 8.1$ & $\begin{array}{l}\text { Zúñiga 1996a, Zúñiga- } \\
\text { González 2000, Zúñiga- } \\
\text { González } 2002\end{array}$ \\
\hline Jaguar (Panthera onca) & 2 & $10.0,18.0$ & $14.0 \pm 5.7$ & Zúñiga-González, 2002 \\
\hline Puma (Puma concolor) & 2 & $18.0,19.0$ & $18.5 \pm 0.7$ & Zúñiga-González, 2001b \\
\hline Tigre de Bengala (Panthera tigris) & 8 & $\begin{array}{l}19.0,21.0,23.0,17.0,17.0,23.0 \\
24.0,14.0\end{array}$ & $19.8 \pm 3.6$ & Zúñiga-González, 2001b \\
\hline Ocelote (Felis pardalis) & 3 & $13,37.0,23.0$ & $24.5 \pm 11.8$ & Zúñiga-González, 2000 \\
\hline Leopardo (Panthera pardus) & 1 & 57.0 & $57.0 \pm 0.0$ & Zúñiga-González, 2002 \\
\hline
\end{tabular}

Los valores se indican como promedio \pm desviación estandar (DE) de EMNe en 10,000 eritrocitos. n: número de individuos muestreados; EMNe: eritrocitos micronucleados espontáneos. 
dentro de una familia. En otras palabras, cuando observamos algún individuo de este grupo no queda duda de que se tiene a un felino (BlankHamer 1983, Vaughan 1988).

Si bien, sólo hemos podido muestrear aproximadamente la cuarta parte de las especies existentes y no tenemos un representante de cada especie, nosotros presuponemos que el resto también presentará EMNe, esto lo fundamentamos en que todas las especies aquí muestreadas presentaron EMNe y es notorio que son especies originarias de diferentes partes del mundo, con lo que queda claro que el origen de esta familia se dio a partir de un ancestro común que originó a los demás géneros, incluidos a los actuales y por lo mismo, es muy probable que compartan esta característica. Nuestros resultados, por tanto, son acordes con el origen propuesto de esta familia a partir de un ancestro extinto (Vaughan 1988). Por tanto, si el origen fue de esta manera, este ancestro debió tener un sistema de limpieza de EMN poco eficiente, el cual fue heredado a todas las especies de esta familia. Desde un punto de vista evolutivo y de adaptación, alguna ventaja les dio, ya que esta característica se ha mantenido a través del tiempo, y se ha conservado en las especies actuales.

Este hallazgo nos plantea dos preguntas inmediatas:

\section{¿Las especies que conforman a esta familia podrían ser potenciales monitores para estudiar genotóxicos?}

Este cuestionamiento lo pudiéramos contestar parcialmente si tomamos en cuenta que las especies con EMN espontáneos han sido las de utilidad para realizar la prueba de $\mathrm{MN}$, como es el caso del ratón, el hámster o la ardilla (Hart 1983, Hayashi 1990, Zúñiga-González 2001a), Si bien, más importante puede resultar el antecedente de que el gato ya ha sido demostrado como un potencial bioindicador (Zúñiga-González 1998), con la ventaja de que, por un lado, es una especie territorial, lo que permitirá realizar trabajos de monitoreo de zonas delimitadas al aportar datos del lugar en el que habita, o por otro lado, puede ser utilizado en laboratorio, como ya históricamente ha sucedido. En el presente otras especies de felinos, como el gato siamés, lince, jaguar, puma, tigre de bengala, ocelote y leopardo, presentan incluso valores más altos que el gato doméstico, por lo que son potencialmente factibles de ser considerados.

\section{¿Cuál es la causa de que este grupo presente EMN espontáneos, cuando lo común es que éstos sean retirados de la circulación rápidamente?}

Este cuestionamiento es más difícil de contestar y sólo podremos lucubrar respecto a datos que conocemos del comportamiento de los EMN. Sabemos que los EMN se presentan cuando el sistema inmune se encuentra inmaduro, como sucede con varias especies incluido el humano (Zúñiga-González 2001b), y que conforme el individuo madura, estos disminuyen hasta quedar en valores cercanos a cero, lo que no es el caso de la familia aquí estudiada. Hemos identificado la importancia del bazo en algunas especies para la eliminación de EMN de la circulación (Ramírez-Muñoz 1999b) y también hemos podido relacionar la presencia de estas estructuras en organismos inmunológicamente inmaduros (Zúñiga-González 2001b), de hecho los felinos, como otras especies, tienen valores más altos de EMN en los organismos con pocos días de nacidos que en individuos adultos. Sin embargo, el encontrar EMN en los felinos no podemos interpretarlo como un problema inmunológico de la familia, ni pensar que las especies con EMN espontáneos serán más susceptibles a presentar infecciones por tener menor eficiencia de sus sistemas de "limpieza". Sin embargo, desde éste punto de vista resulta que sí se observa este fenómeno, pero sólo es real en organismos jóvenes y no así en estado adulto. Los valores de EMN espontáneos son característicos de cada especie y su presencia pareciera estar relacionada exclusivamente con la capacidad que tiene el sistema reticuloendotelial para retirarlos de la circulación más rápidamente o en su totalidad 
en algunos casos o más tardíamente y permitir su circulación en otros casos.

Algo relacionado también lo vemos con el humano en el que, sólo se pueden observar EMN espontáneos en recién nacidos inmaduros (Zúñiga-González 2001b) o cuando las personas son esplenectomizadas (Schlegel 1986, Corazza 1990, Zúñiga 1996b), lo que también ocasiona una menor capacidad de respuesta por parte del sistema inmune del individuo.

De cualquier manera, la presencia de EMN espontáneos en los felinos es la característica que se requiere para realizar la prueba de $\mathrm{MN}$ en eritrocitos de sangre periférica y nos da la posibilidad de utilizarlos como bioindicadores de genotóxicos, básicamente micronucleogénicos.

La prueba de MN que se pretende utilizar en estas especies puede resultar una de las pruebas más sencillas y económicas descritas hasta ahora, ya que sólo se requiere una gota de sangre, portaobjetos, colorantes y un microscopio, por lo que es una técnica al alcance incluso del laboratorio más modesto.

Contar con organismos exóticos con posibilidades para detectar genotóxicos ambientales, además de la alternativa de trabajar con ellos en el laboratorio, nos permite tener opciones para estudiarlos en su medio natural y con esto, conocer lo contaminado que éste pudiera encontrarse, sólo basta elegir correctamente la especie a utilizar.

En el presente trabajo mostramos que la familia de los felinos, debido a sus características particulares de tener EMN espontáneos, son potenciales indicadores de contaminación que afecta directamente el material genético, evidenciado de manera contundente mediante la prueba de $\mathrm{MN}$.

\section{RESUMEN}

La prueba de micronúcleos (MN) en sangre periférica es rápida, sencilla, económica y sirve para detectar genotóxicos ambientales. Los MN son fragmentos de cromosomas o cromosomas completos que por alguna causa quedan fuera del núcleo en mitosis, pero que incrementan significativamente cuando los organismos que los presentan de manera espontánea se exponen a genotóxicos. Por lo tanto, el requisito para que una especie pueda ser utilizada para esta prueba es que presente eritrocitos micronucleados espontáneos (EMNe), con lo que estas especies pueden ser potenciales bioindicadores de genotóxicos micronucleogénicos, con sólo una gota de su sangre. En el presente articulo es mostramos 9 especies de felinos que como característica general presentan EMNe. Del total de especies de felinos, el gato ha sido previamente probado, con resultados positivos y ya que también el león, yaguaroundi, lince, jaguar, puma, tigre de bengala, ocelote y leopardo presentan EMNe, esta familia puede ser propuesta como un grupo potencialmente adecuado para estudios de toxicogenética. En otras palabras, cada una de estas especies puede llegar a ser un modelo potencial para determinar exposición a genotóxicos en nuestro entorno, de una manera sencilla y rápida.

Palabras clave: Felinos, micronúcleos, bioindicador, genotoxicidad, eritrocitos, sangre periférica.

\section{REFERENCIAS}

Afshari, A., P. McGregor, J. Allen \& J. Fuscoe. 1994. Centromere analysis of micronuclei induced by 2-aminoanthraquinone in cultured mouse splenocytes using both a gamma -satellite DNA probe and antikinetochore antibody. Environ. Mol. Mutagen. 24: 96-102.

Blank-Hamer, I.J. 1983. El maravilloso mundo de los gatos. México: Continental.

Corazza, G., L. Ginaldi, G. Zoli, M. Frisoni, G. Lalli, G. Gasbarrini \& D. Quaglino. 1990. Howell-Jolly body counting as a measure of splenic function, a ressesment. Clin. Lab. Haematol. 12: 269-275.

Hart, J. \& B. Hartley-Asp. 1983. Induction of micronuclei in the mouse: revised timing of the final stage of erythropoiesis. Mutat. Res. 120: 127-132.

Hayashi, M., T. Morita, Y. Kodama, T. Sofuni \& M. Ishidate. 1990. The micronuclei assay with mouse peripheral blood reticulocytes using acridine orangecoated slides. Mutat. Res. 245: 245-249.

Heddle, J., C. Lue, E. Saunder \& R. Benz. 1978. Sensitivity to five mutagens in Fanconi's anemia as a measure by the micronucleus method. Cancer. Res. 38: 2983-2988.

Heddle, J.A., M. Hite, B. Kirkhart, K. Mavournin, J.T. MacGregor, G.W. Newell \& M.F. Salamone. 1983. The induction of micronuclei as a measure of genotoxicity. A report of the U.S. Environmental Protection Agency Gene-tox Program. Mutat. Res. 123: 61-118. 
McDonald, G.A., J. Paul \& B. Cruickshank. 1998. Atlas de Hematología. Panamericana, Madrid, España.

Oxenham, M. 1999. Hurones, p. 113. In P.H. Beynon \& J.E. Cooper (eds.). Manual de Animales Exóticos. Hartcourt Brace, Madrid, España.

Ramírez-Muñoz, M.P., O. Torres-Bugarín, A. Ramos, J. Sánchez-Corona \& G. Zúñiga. 1999a. Evidencia de micronucleogenicidad especie-específica del cloramfenicol en roedores de laboratorio. AMMVEPE 10: 50-52.

Ramírez-Muñoz, M.P., G. Zúñiga, O. Torres-Bugarín, E. Portilla, D. García-Martínez, A. Ramos, J.M. Cantú \& J. Sánchez-Corona. 1999. Evaluation of the micronucleus test in peripheral blood erythrocytes by use of the splenetomized model. Lab. Anim. Sci. 49: 418-420.

Rodríguez-Ariza, A., A. Nieves, J.I. Navas, G. Dorado, J. López-Barea \& C. Pueyo. 1992. Metal mutagenicity and biochemical studies on bivalve molluscs from Spanish coasts. Environ. Mol. Mutagen. 19: 112-124.

Russo, A. \& A.G. Levis. 1992. Further evidence for the aneuploidogenic properties of chelating agents: induction of micronuclei in mouse male germ cells by EDTA. Environ. Mol. Mutagen. 19: 125-131.

Schlegel, R., J. MacGregor \& R. Everson. 1986. Assesment of cytogenetic damage by quantitation of micronuclei in human peripheral blood erythrocytes. Cancer. Res. 46: 3717-3721

Schmezer, P., B.L. Pool, P.A. Lefevre, R.D. Callander, F. Ratpan, H. Tinwell \& J. Ashby. 1990. Assay-Specific genotoxicity of n-nitrosodibenzylamine to the rat liver in vivo. Environ. Mol. Mutagen. 15: 190-197.

Schmid, W. 1975. The micronucleus test. Mutat. Res. 31: 9-15.

Vaughan, T.A. 1988. Mamíferos. McGraw Hill, México D.F., México.

Yamamoto, K.I. \& Y.A. Kikuchi. 1980. Comparison of diameters of micronuclei induced by clastogens and by spindle poisons. Mutat. Res. 71: 127-131.

Zúñiga, G., O. Torres-Bugarín, M.P. Ramírez-Muñoz, A. Ramos, E. Fanti-Rodríguez, E. Portilla, D. GarcíaMartínez, J.M. Cantú, M.P. Gallegos-Arreola \& J. Sánchez-Corona. 1996a. Spontaneous micronuclei in peripheral blood erythrocytes from 35 mammalian species. Mutat. Res. 369: 123-127.

Zúñiga, G., O. Torres-Bugarín, M.P. Ramírez-Muñoz, J.L. Delgado-Lamas, R. De Loza-Saldaña \& J.M.
Cantú. 1996b. Micronucleated erythrocytes in splenectomized patients with and without chemotherapy. Mutat. Res. 361: 107-112.

Zúñiga-González, G., B.C. Gómez-Meda, A. ZamoraPerez, M.L. Ramos-Ibarra, C.M. Batista-González, A. González-Rodríguez, J. Luna-Aguirre \& J.L. Rodríguez-Ávila. 2002. Especies exóticas con micronúcleos espontáneos: alternativa para estudios de genotoxicidad. Nowet. 1: 5-9.

Zúñiga-González, G., B.C. Gómez-Meda, A. ZamoraPerez, M.L. Ramos-Ibarra, C.M. Batista-González, S. Espinoza-Jiménez, M.P. Gallegos-Arreola, C. Álvarez-Moya \& O. Torres-Bugarín. 2003. Induction of micronuclei in proestrus vaginal cells from colchicine- and cyclophosphamide-treated rats. Environ. Mol. Mutagen. 42: 306-310.

Zúñiga-González, G., M.P. Ramírez-Muñoz, O. TorresBugarín, J. Pérez-Jiménez, A. Ramos-Mora, A. Zamora-Perez, M.P. Gallegos-Arreola \& J. SánchezCorona. 1998. Induction of micronuclei in the domestic cat (Felis domesticus) peripheral blood by colchicine and cytosine-arabinoside. Mutat. Res. 413: 187-189.

Zúñiga-González, G., O. Torres-Bugarín, J. Luna-Aguirre, A. González-Rodríguez, A. Zamora-Pérez, B.C. Gómez-Meda, A.J. Ventura-Aguilar, M.L. RamosIbarra, A. Ramos-Mora, G.G. Ortiz, M.P. GallegosArreola. 2000. Spontaneous micronuclei in peripheral blood erythrocytes from 54 animal species (mammals, reptiles and birds): Part two. Mutat. Res. 467: 99-103.

Zúñiga-González, G., O. Torres-Bugarín, M.L. RamosIbarra, A. Zamora-Perez, B.C. Gómez-Meda, A.J. Ventura-Aguilar, A. Ramos-Mora, G.G. Ortiz, C. Álvarez-Moya, A. González-Rodríguez, J. LunaAguirre \& M.P. Gallegos-Arreola. 2001a. Variation of micronucleated erythrocytes in peripheral blood of Sciurus aureogaster in relation to age: an increment of micronucleated polychromatic erythrocytes after the administration of colchicine. Environ. Mol. Mutagen. 37: 173-177.

Zúñiga-González, G., O. Torres-Bugarín, A. ZamoraPerez, B.C. Gómez-Meda, M.L. Ramos-Ibarra, S. Martínez-González, A. González-Rodríguez, J. LunaAguirre, A. Ramos-Mora, D. Ontiveros-Lira \& M.P. Gallegos-Arreola. 2001b. Differences in the number of micronucleated erythrocytes among young and adult animals including humans. Spontaneous micronuclei in 43 species. Mutat. Res. 494: 161-167. 